\title{
Optical properties of Au nanostructures obtained by pulsed UV laser irradiation of thin films
}

\author{
K. Grochowska* and G. Śliwiński \\ Photophysics Dept., The Szewalski Institute of F-FM, Polish Academy of Sciences, 14 Fiszera St, 80-231 Gdansk
}

Received May 13, 2011; accepted August 31, 2011; published September 30, 2011

\begin{abstract}
The particle size distribution, morphology and optical properties of the Au nanoparticle structures are investigated in relation to their preparation conditions. The structures are produced from thin $\mathrm{Au}$ films (10-20nm) sputtered on $\mathrm{SiO}_{2}$ glass substrate and annealed with several pulses (6ns) of laser radiation at $266 \mathrm{~nm}$ and at fluencies in the range of $10-400 \mathrm{~mJ} / \mathrm{cm}^{2}$. The scanning electron microscopy (SEM) inspection reveals nearly homogeneously distributed, spherical gold particles with an average radius of $40 \mathrm{~nm}$. In the absorbance spectra of the nanostructures the broad peak centered at $550 \mathrm{~nm}$ is ascribed to resonant absorption of surface plasmons (SPR). The peak position, halfwidth and intensity depend on the shape, size and size distribution of the nanostructured particles in agreement with literature. From peak intensities of the Raman spectra, recorded for Rhodamine $6 \mathrm{G}$ in the range of $300-1800 \mathrm{~cm}^{-1}$, the relative signal enhancement for individual peaks (by a factor between 20 and 603) is estimated. Results confirm that the obtained structures are suitable for surface enhanced Raman spectroscopy (SERS) measurements and sensing.
\end{abstract}

During the last two decades the phenomenon of surface plasmon resonance (SPR) has been intensively studied. It has found its practical applications in detectors capable of detecting single molecules. The SPR effect can be obtained for metal particles of dimensions much smaller than the wavelength of interacting light [1]. In the case of homogenously distributed spherical particles their optical properties can be derived from the Mie theory. For very small particles of radius $r$ (where $2 r<<\lambda$ and $\lambda$ is a wavelength of light), the extinction coefficient $\alpha$ can be expressed as:

$$
\alpha=18 \pi \mathrm{r}^{3} \mathrm{NV} \frac{1}{\lambda} \frac{\varepsilon_{2}}{\left(\varepsilon_{1}+2 \mathrm{n}^{2}\right)^{2}+\varepsilon_{2}^{2}}
$$

where $\varepsilon=\varepsilon_{1}(\lambda)+\mathrm{i} \varepsilon_{2}(\lambda)$ is the wavelength-dependent, complex dielectric function of the nanoparticle (NP) material with $\varepsilon_{1}=10-58 \lambda^{2}$ and $\varepsilon_{2}=0.1+3.4\left(1_{0} / \mathrm{r}+1\right) \lambda^{3}$; $\mathrm{n}$ - the refractive index of the surrounding/embedding medium, $\mathrm{N}$ - the number of particles per unit volume, $1_{0}-$ the mean free path of electrons in the bulk and $\mathrm{V}-$ the volume of a single particle [2]. In this work we use the above approach to describe and discuss the properties of nanostructures produced by means of pulsed UV laser irradiation of thin Au films.

\footnotetext{
*E-mail: kgrochowska@imp.gda.pl
}

Thin Au films of 10-20nm thickness on glass substrate were produced from bulk material (Sigma Aldrich; 99.99\% purity) by RF discharge sputtering at an ambient pressure of $4 \cdot 10^{-2} \mathrm{mbar}$. The annealing of the films for the production of NP structures was performed in vacuum $\left(10^{-6} \mathrm{mbar}\right)$ by pulsed (pulse duration: 6ns, pulse frequency: $2 \mathrm{~Hz}$ ) radiation of the $\mathrm{Nd}$ :YAG laser (Quantel B) operated at $266 \mathrm{~nm}$ and at fluencies in a range of 10 $400 \mathrm{~mJ} / \mathrm{cm}^{2}$. Up to 15 pulses were applied per sample at selected laser fluencies obtained by changing the dimension of a laser spot.

SEM inspection was performed by means of the EVO40 microscope (Zeiss) and recording of the $\mu$ Raman spectra was provided by the InVia confocal instrument (Renishaw) equipped with a $785 \mathrm{~nm}$ laser for sample excitation.

For absorbance measurements of the Au NP structures the spectrophotometer UV 1240 (SHIMADZU) was applied.

Thin film samples deposited by discharge sputtering reveal no defects. The fluence values of $160-412 \mathrm{~mJ} / \mathrm{cm}^{2}$ applied during laser irradiation of $10 \mathrm{~nm}$ and $15 \mathrm{~nm}$ thick $\mathrm{Au}$ films on glass result in the nanostructured samples shown in Figs. 1-2. A nearly homogeneous distribution of spherical particles is characteristic for all investigated structures, and this is consistent with our previous work $[3,4]$. Depending on the pulse number applied, the particle number per unit area decreases with prolonged irradiation at fixed laser fluence of $412 \mathrm{~mJ} / \mathrm{cm}^{2}$, see Figs. 1a-c. A low irradiation dose of 5 pulses results in a relatively narrow size distribution of NPs in a range of $20-80$ nm with a prevailing number of small ones of a diameter between $20 \mathrm{~nm}$ and $40 \mathrm{~nm}$. After the next 5 pulses the number of the smallest particles decreases markedly and the average particle diameter is estimated to be greater than $80 \mathrm{~nm}$. After further irradiation the total particle number decreases.

The measured absorption spectrum of the nanostructures produced by annealing films of an original thickness of $10 \mathrm{~nm}$ is shown in Fig. 3. The broad peak of the profile centered around 550nm corresponds to the 
resonant absorption of surface plasmons and is red-shifted relative to the resonance position of a single Au molecule [5]. It can be expected that the profile and peak position of the measured SPR band depends on the number, shape and size of the nanoparticles.

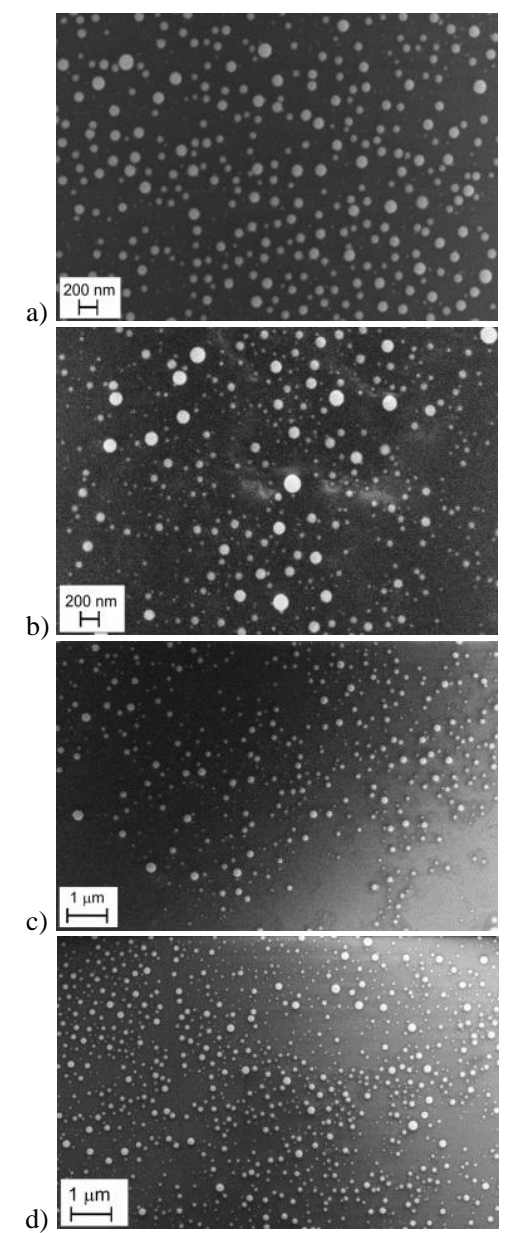

Fig. 1. SEM images of Au nanoparticles obtained from the films of a thickness of d 10nm, irradiated with 5 (a), 10 (b), 15 (c) laser pulses at $266 \mathrm{~nm}$ and a fixed fluence of $412 \mathrm{~mJ} / \mathrm{cm}^{2}$ and with 15 (d) pulses at $160 \mathrm{~mJ} / \mathrm{cm}^{2}$

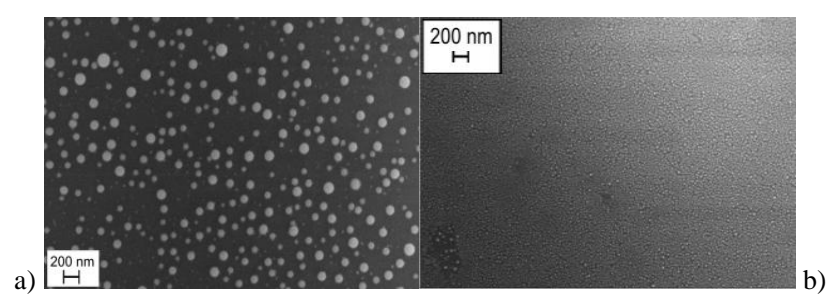

Fig. 2. SEM images of Au nanoparticles obtained from the films of a thickness of d 10nm (a) and $15 \mathrm{~nm}$ (b), irradiated with 5 (a) and 10 (b), laser pulses at $266 \mathrm{~nm}$ and a fixed fluence of $412 \mathrm{~mJ} / \mathrm{cm}^{2}$.

The absorption spectra confirm that the $\mathrm{Au}$ nanoparticles are indeed spherical in shape. Only a single plasmon band is observed in obtained spectra. Therefore the profile and position of the plasmon absorption peak can be described by using the Mie absorption and scattering theory, Eq. (1). The normalized spectral profile calculated for $1_{0}=20 \mathrm{~nm}[6]$ and for a particle dimension of $20 \mathrm{~nm}$ is shown as spectrum d2 in Fig. 3.

From the resonance position the particle radius can be calculated. In the range of a particle diameter of 20 $160 \mathrm{~nm}$ the value of $\mathrm{r}$ can be expressed as:

$$
\mathrm{r}=107.5 \sqrt{2.262-\mathrm{E}_{\text {res }}}
$$

where $\mathrm{E}_{\text {res }}$ is the resonance energy given in $\mathrm{eV}$ [7].

For spectra shown in Fig. 3, the resonance energy of $2.27 \mathrm{eV}$ follows and the average particle diameter of 20 $40 \mathrm{~nm}$ is estimated basing on data shown on Fig. 4. This result agrees with our expectation.

From the homogenous linewidth of the absorption profile the dephasing time can be estimated:

$$
T_{2}=\frac{\Gamma}{2 \hbar}
$$

where $\Gamma$ is the full linewidth at half maximum (FWHM) of plasmon resonance. The dephasing time describes the amount of damping of plasmon radiation. Dephasing times calculated for spectra shown in Fig. 3 are presented in Tab. 1.

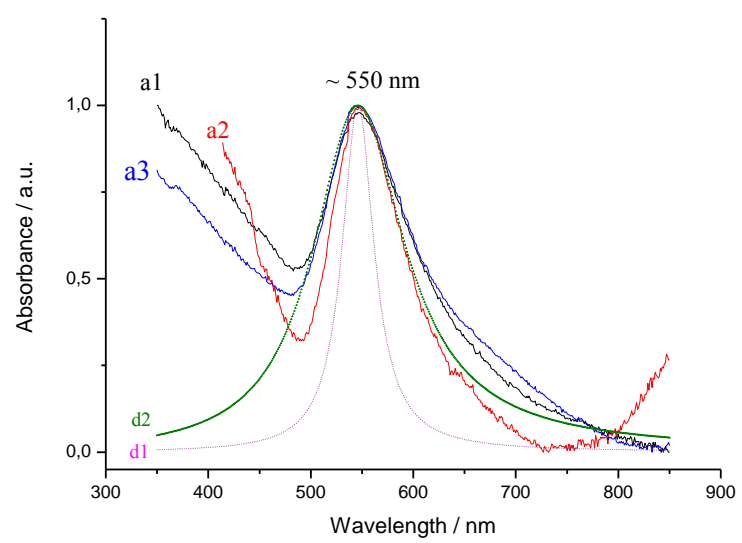

Fig. 3. Absorbance spectra of the Au nanoparticle structures on $\mathrm{SiO}_{2}$ substrate; profile a 2 corresponds to the structure shown in Fig. 2 b; a1, a3 - structures produced by 5 and 15 laser pulses under the same conditions as in Fig. 2 b; profiles (d1) and (d2) are calculated for a particle dimension of $4 \mathrm{~nm}$ and $20 \mathrm{~nm}$.

Tab. 1. The dephasing times calculated for profiles shown in Fig. 3.

\begin{tabular}{|l|c|c|}
\hline $\begin{array}{l}\text { profil } \\
\text { e }\end{array}$ & FWHM [eV] & $\mathbf{T}_{\mathbf{2}}[\mathbf{f s}]$ \\
\hline $\boldsymbol{a} \mathbf{1}$ & 0.59838 & 2.199 \\
\hline $\boldsymbol{a} \mathbf{2}$ & 0.41461 & 3.175 \\
\hline $\boldsymbol{a 3}$ & 0.60803 & 2.165 \\
\hline
\end{tabular}




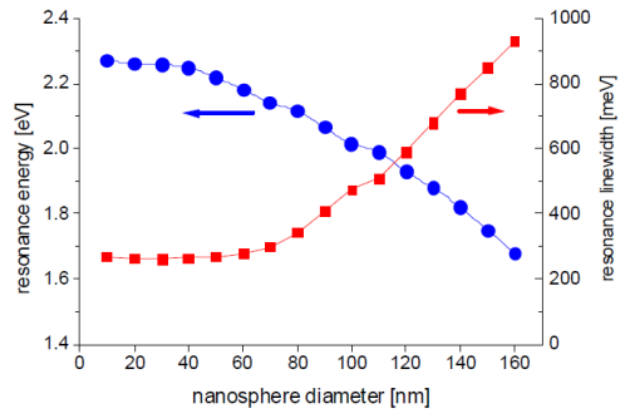

Fig. 4. The resonance position and spectral linewidth of gold nanoparticles vs the particle size calculated from the Mie theory [7].

For measurement of the surface enhanced Raman (SER) signal, the spectra of samples covered with dried solution of the $0.09 \mathrm{M}$ Rhodamine G6 are recorded under mild excitation at $785 \mathrm{~nm}$, which should provide efficient near field enhancement at a strong Raman signal, in agreement with numerical simulation and literature data [4, 8, 9]. Figure 5 shows non-corrected Raman spectra of R6G deposited on glass (NR), on 10nm thick Au film on glass (a) and on the nanostructured gold substrates (b), (c) presented in Figs. 1d and 1a.

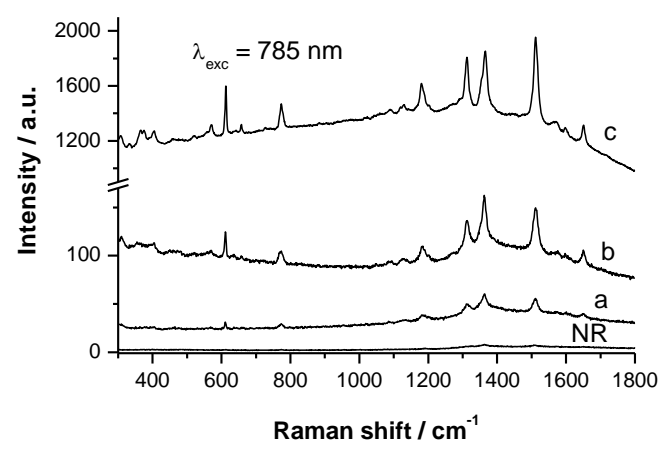

Fig. 5. Comparison between the Raman spectra of R6G deposited on: glass- NR (from [12]), (a) - Au non-structured film, and (b), (c) - gold nanoparticle structures shown in Figs. 1d and 1a, respectively; samples excited at $\lambda=785 \mathrm{~nm}$.

Direct comparison of the peak intensities of spectrum (NR) with these of (b) and (c) in the range of 600 $1550 \mathrm{~cm}^{-1}$ shows relative enhancement of the Raman signal by a factor varying between 20-48 and 239-603 for individual peaks of spectra (b) and (c), respectively.
This result is satisfactory because the properties of nanostructure discussed here in part only match these of large SERS ensured in interstices between nanoparticles smaller than reported here and with distances of less than $5 \mathrm{~nm}$, where the intensity can build up by several orders of magnitude.

In conclusion, for thin (10-20 nm) Au films sputtered on glass and annealed by pulsed UV laser radiation the nearly homogeneous nanostructures of spherical gold particles and narrow particle size distribution were prepared.

It was observed that the absorbance profile and the resulting dephasing time depend strongly on substrate preparation conditions. Changes of the dephasing time in a range of 2.2-3.1fs were obtained from absorbance spectra of the nanostructured Au/glass substrates. The decrease of dephasing time with increasing particle size and size distribution is accompanied by a slight red shift of the absorbance profile.

The effect observed in the experiment can be exploited in the SERS-based biosensor research. Larger enhancement of the Raman signal is expected for smaller particles forming closely packed structures of controlled geometry and work on this topic is in progress.

The work was supported by the strategic program of scientific research and experimental development of the National (Polish) Centre for Research and Development: "Advances Technologies for Energy Generation"; Task 4. "Elaboration of Integrated Technologies for the Production of Fuels and Energy ...". The technical assistance of Dr. R. Signerski, Dr. M. Sawczak and Mr A. Sobczyk is gratefully acknowledged.

\section{References}

[1] U. Kreibig, M. Vollmer, Optical properties of metal clusters (Berlin, Springer 1995).

[2] J. Vosburgh, R.H. Doremus, J. Non-Cryst. Sol. 349, 309 (2004).

[3] S.E. Imamova, A. Dikovska, N.N. Nedyalkov, P.A. Atanasov, M. Sawczak, R. Jendrzejewski, G. Sliwinski, M. Obara, J. Optoel. Adv. Mat. 12, 500 (2010).

[4] N.N. Nedyalkov, S.E. Imamova, P.A. Atanasov, M. Obara, Appl. Surf. Sci. 255, 5125 (2009).

[5] M.M. Alvarez, J.T. Khoury, T.G. Schaaff, M.N. Shafigullin, I. Vezmar, R.L. Whetten, J. Phys. Chem. B 101, 3706 (1997).

[6] M.L. Theye, Phys. Rev. B 2, 3060 (1970).

[7] C. Sonnichsen, Plasmons in metal nanostructures (University of Munich 2001).

[8] G. Li, H. Li, Y. Mo, X. Huang, and L. Chen, Chem. Phys. Lett. 330, 249 (2000).

[9] M. Michaels, J. Jiang, L. Brus, J. Phys. Chem. B 104, 11965 (2000). 